\title{
Adaptation of endothelial cells to shear stress under atheroprone conditions by modulating internalization of vascular endothelial cadherin and vinculin
}

\author{
Tingting Zhong ${ }^{1,2}$, Yanling $\mathrm{Li}^{1,3}$, Xiaohong $\mathrm{He}^{1}$, Yongdong Liu ${ }^{4}$, Yugang Dong ${ }^{1}$, Hong $\mathrm{Ma}^{1}$, \\ Zhensheng Zheng ${ }^{1}$, Yan Zhang ${ }^{1}$
}

${ }^{1}$ Department of Cardiology, NHC Key Laboratory of Assisted Circulation, First Affiliated Hospital, Sun Yat-sen University, Guangzhou, China; ${ }^{2}$ Department of Cardiology, Fuwai Hospital, Chinese Academy of Medical Sciences, Shenzhen, China; ${ }^{3}$ Department of Emergency, Third Affiliated Hospital, Sun Yat-sen University, Guangzhou, China; ${ }^{4}$ Department of Pathology, First Affiliated Hospital, Sun Yat-sen University, Guangzhou, China

Contributions: (I) Conception and design: Y Zhang, T Zhong; (II) Administrative support: Y Dong, H Ma, Z Zheng; (III) Provision of study materials or patients: Y Zhang, T Zhong, Y Li, X He, Y Liu; (IV) Collection and assembly of data: T Zhong, Y Li, X He; (V) Data analysis and interpretation: Y Zhang, T Zhong, Y Li, Y Liu; (VI) Manuscript writing: All authors; (VII) Final approval of manuscript: All authors.

Correspondence to: Yan Zhang, MD. Division of Cardiovascular Rehabilitation, Department of Cardiology, NHC Key Laboratory of Assisted

Circulation, First Affiliated Hospital, Sun Yat-sen University, 58 Zhongshan Road, Guangzhou, China 510080, China.

Email: zhyan3@mail.sysu.edu.cn; gzyanzhang@163.com.

Background: Endothelial cells play a pivotal role in cardiovascular physiology and pathology by providing a barrier to the bloodstream. In the current study, we investigated the phenotype and barrier function of endothelial cells in response to shear stress under pro-atherogenic conditions.

Methods: Endothelial cells were exposed to laminar shear stress (LSS) in a parallel-plate flow chamber containing oxidized low-density lipoprotein (oxLDL) in the perfusion solution, or remained static. We quantified the response of endothelial monolayers to LSS and oxLDL in terms of cell viability, barrier integrity, vascular endothelial cadherin (VE-cadherin) availability, focal adhesion (FA) remodeling, and monocyte-endothelial interactions.

Results: Our results showed that oxLDL stimulation and static conditions synergized to enhance endothelial barrier disruption. Under the same oxLDL challenge, the application of $25 \mathrm{dynes} / \mathrm{cm}^{2} \mathrm{LSS}$ on the endothelial monolayer decreased the passage of fluorescein isothiocyanate (FITC)-dextran by $37.79 \%$, increased transendothelial electrical resistance (TEER) by $24.97 \%$ compared with static cells $(\mathrm{P}<0.05)$, which was accompanied by reduced intercellular gap formation, relatively solid cell-substrate adhesion. Compared with static cells, endothelial cells exposed to both laminar flow and oxLDL had less small FAs, less monocyte adhesion and transmigration, and alleviated overexpression of VCAM-1 and MCP-1. Meanwhile, the oxLDL-induced internalization of VE-cadherin and vinculin were also attenuated by laminar flow, and this change was more pronounced at LSS of 25 dynes $/ \mathrm{cm}^{2}$ than 5 dynes $/ \mathrm{cm}^{2}$.

Conclusions: Static conditions favor, whereas physiologically higher levels of LSS ameliorate endothelial barrier disruption under pro-atherogenic stress, which is related to the improved availability of VE-cadherin and vinculin on the cell surface.

Keywords: Atherosclerosis; endothelial cells; shear stress; focal adhesion; endothelial barrier dysfunction

Submitted Apr 20, 2020. Accepted for publication Aug 28, 2020.

doi: $10.21037 / \mathrm{atm}-20-3426$

View this article at: http://dx.doi.org/10.21037/atm-20-3426 


\section{Introduction}

Cardiovascular disease is currently the leading cause of death worldwide. Studies on human arteries have demonstrated that atherosclerosis is the most crucial underlying pathology of cardiovascular disease. Vascular smooth muscle $\alpha$-actin-positive resident cells and macrophages are now considered to be the two most significant sources of foam cells. The majority of lipids during foam cell formation come from modified lowdensity lipoprotein (LDL), not native LDL. LDL undergoes multiple post-translational modifications (such as oxidation and glycosylation) and acquires atherogenic properties $(1,2)$. The endothelium serves as a semi-selective barrier between the bloodstream and surrounding tissues, and controls the exchange of proteins, solutes, and liquid, as well as the migration of cells. If the permselective barrier function of the endothelium is weakened, molecules, such as modified LDL, and monocytes will enter the subendothelial space via circulation to initiated chronic inflammation and immune disorders. According to current consensus, the primary event in atherosclerotic lesion development is local endothelial activation and permeability enhancement, which may be associated with hemodynamic forces occurring at the sites prone to atherosclerosis (3-5).

It has been reported that atherosclerotic plaques preferentially develop at the lateral wall of the vascular bifurcations, the branch points, and the lesser curvature of the aortic arch, which are the sites of blood flow recirculation with low or oscillating shear stress (6). Hemodynamic shear stress, the frictional force acting on the endothelial surface as a result of blood flow, plays a vital role in the development of atherosclerosis. Low shear stress $\left(<4\right.$ dynes $\left./ \mathrm{cm}^{2}\right)$ has been shown to promote atherosclerosis, while normal-to-high shear stress $\left(>15\right.$ dynes $\left./ \mathrm{cm}^{2}\right)$ protects against atherosclerosis in humans $(7,8)$. Vascular endothelial cells (VEC) respond to sustained laminar shear stress (LSS) by increasing the production of vasodilators and antioxidants, reducing the expression of inflammatory mediators and growth factors $(9,10)$.

The integrity of the endothelial barrier depends on the adhesion between adjacent endothelial cells and the adhesion of endothelial cells to the underlying matrix. Adherens junctions are specialized sites for cell-cell adhesion, and VE-cadherin is the primary adhesion molecule. Endocytosis of VE-cadherin regulates junctional strength, and the reduction of membrane-bound VEcadherin leads to vascular destabilization, intercellular gap opening and loss of barrier function (11). Focal adhesions (FAs) are another critical junction regulating endothelial barrier function. As the structures connecting the actin cytoskeleton with the extracellular matrix, FAs physically connect the external environment to the interior of the cell through the actin cytoskeleton $(12,13)$.

Although intense efforts have been made to understand how cells sense and respond to the properties of the micro-environment, the underlying mechanisms for their function are not fully understood. Therefore, our research aimed to gain a deeper understanding of the biological characteristics of VECs and the pathological basis of atherosclerosis. We explored the behavior of VEC cultured under static or constant laminar flow conditions while challenged by pro-atherogenic oxidized LDL (oxLDL). We hypothesized that an appropriate increase in LSS would ameliorate the deleterious effects of oxLDL on the vascular endothelium. Our results provide new insights into the regulation of vascular endothelial integrity and highlight the role of shear stress in maintaining endothelial barrier function. In the adaptation process of endothelial cells to microenvironmental stimuli such as shear stress and oxLDL, in addition to changes in content, the availability of VE-cadherin and vinculin on the cell surface is also crucial.

\section{Methods}

\section{Endothelial cell culture and intervention}

Human umbilical endothelial cells (HUVEC) were isolated from fresh normal-term human umbilical cords using collagenase perfusion technique (14). Experiments were performed with four-to-five passages of cells. HUVEC $\left(1 \times 10^{5}\right.$ cells $\left./ \mathrm{cm}^{2}\right)$ were seeded onto the $\mu$-slides of parallel plate flow chambers (ibidi, Munich, Germany) to attain confluence 24 hours before flow applications, as described previously $(15,16)$.

An ibidi pump system (ibidi, Germany) was used to generate unidirectional flow across the endothelial monolayer at a constant LSS of 5 or 25 dyne $/ \mathrm{cm}^{2}$ for 4 hours. The chambers and reservoirs containing the medium were kept in an incubator at $37{ }^{\circ} \mathrm{C}$ and $5 \% \mathrm{CO}_{2}$. To mimic atheroprone conditions, human oxLDL (Yiyuan Biotechnologies, Guangzhou, China) was added into the perfusion medium at a concentration of $100 \mu \mathrm{g} / \mathrm{mL}$ (static + oxLDL group, 5 dyne $/ \mathrm{cm}^{2}+$ oxLDL group, 25 dyne $/ \mathrm{cm}^{2}$ + oxLDL group) (17). No flow and no oxLDL conditions served as the control (static group). 


\section{Measurement of transendothelial electrical resistance (TEER)}

TEER was measured using an electrical cell-substrate impedance sensor (ECIS) system (Applied BioPhysics, Troy, NY, USA), as previously described. Data were normalized to baseline resistance to evaluate the integrity of endothelial monolayers $(18,19)$.

\section{Endothelial permeability assay}

Endothelial permeability was evaluated by measuring the passage of fluorescein isothiocyanate-dextran (FITC)dextran (40,000 Da, Sigma-Aldrich, St Louis, MO, USA) through the endothelial monolayer with the use of $\mu$-slide membrane ibiPore $0.5 \mu \mathrm{m} / 20 \%$ flow units (ibidi, Germany) coated with fibronectin $(10 \mu \mathrm{g} / \mu \mathrm{L})$, connected with the ibidi pump system through a perfusion set.

HUVECs were seeded on the lower side of the porous membrane and were allowed to grow to a confluence monolayer. FITC-Dextran $(1 \mathrm{mg} / \mathrm{mL})$ was added directly to the lower channel. HUVECs were perfused in the lower chamber for flow applications for 4 hours, containing oxLDL in the perfusion medium, as previously described. Passive diffusion of the dextran through the monolayer to the upper channel was quantitated by measuring the fluorescence intensity of the medium from each chamber at an excitation peak wavelength of $490 \mathrm{~nm}$ using a SpectraMax microplate fluorometer (Molecular Devices, Sunnyvale, CA, USA). A standard curve of different concentrations of the tracer was utilized to determine the concentration of FITC-dextran in the chambers.

\section{Immunofluorescence staining and image analysis}

HUVEC were incubated with primary antibodies specific for FAK (Rabbit polyclonal antibody, Cell Signaling Technology Cat\# 3285, RRID:AB_2269034), Vascular cell adhesion molecule-1 (VCAM-1) (rabbit monoclonal antibody, Cell Signaling Technology Cat\# 13662, RRID:AB_2798286), integrin $\beta 1$ (rabbit monoclonal antibody, Cell Signaling Technology Cat\# 34971, RRID:AB_2799067) at 1:100 dilution (Cell Signaling Technology, Boston, MA, USA), or vinculin (Rabbit polyclonal antibody, Abcam Cat\# ab73412, RRID:AB_1861566) at 1:200 dilution, VEcadherin (Rabbit polyclonal antibody, Abcam Cat\# ab33168, RRID:AB_870662) at 1:100 dilution (Abcam, Cambridge, MA, USA), or monocyte chemoattractant protein (MCP-1)
(Rabbit polyclonal antibody, Santa Cruz Biotechnology Cat\# sc-28879, RRID:AB_2070877) (Santa Cruz Biotechnology, Santa Cruz, CA, USA) at 1:100 dilution. Immunofluorescence images were obtained with a confocal laser scanning fluorescence microscope (Fluoview FV500IX81, Olympus, Tokyo, Japan) or 780 laser confocal microscope (Carl Zeiss, Jena, Germany).

VE-cadherin disruption was identified through an analysis of the discontinuity of green fluorescence at VE-cadherin junctions between endothelial cells. The interendothelial gap area within disrupted VE-cadherin junctions was determined from five images using ImageJ software, the ratio was quantified as the gap area to the total area of an image.

The size and number of vinculin-containing FAs were quantified using the Analyze Particles function in ImageJ, as described previously $(20,21)$. FA $<0.1 \mu \mathrm{m}^{2}$ were excluded to ensure that background noise was eliminated from the analysis.

\section{Trypsinization experiment}

To exam cell-substrate interactions, endothelial monolayers were trypsinized (Gibco TripLE, Invitrogen, Carlsbad, CA, USA) following the intervention described earlier. The time course of endothelial cell detachment after trypsinization was recorded. The percentage of adherent cells was calculated as follows: (total cell count - detached cell count)/total cell count (22). Light micrograph pictures were acquired using an inverted microscope (DMI8; Leica, Mannheim, Germany).

\section{Flow cytometry analysis}

The expression of integrin $\beta 1$ in HUVECs was quantified by flow cytometry. After intervention, HUVECs were incubated with the PE-Mouse-Anti-Human CD29 (integrin $\beta 1$ ) antibody (BD Biosciences Cat\# 556049, RRID:AB_396320) (BD Biosciences Pharmingen, San Diego, CA, USA ) $(20 \mu \mathrm{L}: 200 \mu \mathrm{L})$ and analyzed using an EPICS XL MCL flow cytometer (Beckman Coulter, Fullerton, CA, USA).

\section{Western blot analysis}

Total protein extracts were prepared using T-PER TM Tissue Protein Extraction Reagent (Thermo Fisher Scientific, Waltham, MA, USA) in the presence of proteinase 
inhibitor mixture (Thermo Fisher Scientific, USA).

Membrane and cytoplasmic protein extracts were prepared using a Mem-PER TM Plus Membrane Protein Extraction Kit (Thermo Fisher Scientific, USA).

For immunoblotting analysis, protein extracts were separated by sodium dodecylsulfate-polyacrylamide gel electrophoresis, transferred to a polyvinylidene difluoride membrane, and probed with specific antibodies at 1:1,000 dilution, as described previously (23).

Reprobing membranes verified equal protein loading with antibody to glyceraldehyde-3-phosphate dehydrogenase (GAPDH) or a specific protein of interest. The signal was detected using an enhanced chemiluminescence detection system (SuperSignal West Pico; Thermo Fisher Scientific, USA).

\section{Monocyte adhesion and transmigration}

HUVECs were seeded on the lower side of the porous membrane of ibiPore flow units and allowed to grow to a confluence monolayer, then perfused in the lower chamber for flow applications, as previously described.

BCECF-AM-labeled quiescent THP- 1 cells $\left(2 \times 10^{4}\right.$ cells $)$ were plated onto the endothelial monolayer and incubated for 24 hours to study the transmigration of THP-1 cells through endothelial monolayer. The THP-1 cells adhering to the monolayer were then observed under a Leica DMI8 inverted microscope. The medium from each chamber was collected, and the THP-1 cells that had transmigrated through the endothelial monolayer to the upper chamber was quantitated by measuring the fluorescence intensity at an optical density of $538 \mathrm{~nm}$ using the SpectraMax microplate fluorometer.

\section{Statistical analysis}

Data are presented as mean \pm standard error of the mean (SEM), unless indicated otherwise. When homogeneity of variance was assessed, statistical significance regarding multigroup comparison was determined by a one-way analysis of variance (ANOVA) followed by the Bonferroni post-hoc test. When variables violated the assumptions for variance homogeneity, significance was alternatively evaluated by the Kruskal-Wallis rank-sum test and MannWhitney post-hoc comparisons. For data of multiple timepoint measurements, the repeated-measures analysis was performed with general linear methods-general factorial ANOVA, followed by the methods above; $\mathrm{P}<0.05$ denoted significant difference. The SPSS 16.0 software (SPSS, Chicago, IL, USA) was used for all statistical calculations.

The study was conducted in accordance with the Declaration of Helsinki (as was revised in 2013). The study was approved by the Ethics Committee of the First Affiliated Hospital, Sun Yat-sen University, China (No. 201060), and informed consent was taken from all the participants.

\section{Results}

\section{Shear stress affects endothelial cell viability and ameliorates endothelial barrier damage evoked by oxidized LDL}

The endothelial monolayer was incubated with the proatherosclerotic factor oxLDL to establish a model of endothelial cell injury while undergoing LSS.

The static cells showed a cobblestone morphology with well-defined cell boundaries based on the phasecontrast image (Figure 1). Under static conditions, oxLDL treatment-induced visible morphologic changes in VECs: deformation, disorder, and shedding; only $75.11 \%$ of VECs remained on the substrate. Although stimulated by oxLDL at the same concentration and duration, the VECs exposed to 25 dynes $/ \mathrm{cm}^{2}$ LSS had a marked shift in cell shape: they became elongated, spindle-like, and tended to be parallel to the flow direction, with a $26.68 \%$ increase in retention rate compared to the static controls $(\mathrm{P}<0.05)$ (Figure 1A,C).

As displayed in Figure $1 D$, the cell counting kit (CCK)- 8 assay indicated that, under static conditions, the administration of oxLDL reduced cell viability by approximately half compared to non-oxLDL controls $(\mathrm{P}<0.05)$; however, this reduction was improved by shear stress application, although these cells were also stimulated by oxLDL $(\mathrm{P}<0.05)$ (Figure $1 D)$.

We then assessed cytotoxicity by measuring lactate dehydrogenase (LDH) release. As shown in Figure1E, under static conditions, the $\mathrm{LDH}$ release level in the ox-LDL treated group was 1.64-fold higher than those without oxLDL $(\mathrm{P}<0.05)$. Endothelial cells simultaneously subjected to shear stress and oxLDL exhibited a dramatic decline in LDH release $(\mathrm{P}<0.05$ vs. static + oxLDL group), and the LDH release level at 25 dyne/ $\mathrm{cm}^{2}$ LSS was $17.99 \%$ lower than at 5 dyne $/ \mathrm{cm}^{2}$ LSS $(\mathrm{P}<0.05)$.

In contrast to the confluent monolayer of the control group retaining its integrity, the oxLDL-attacked monolayer showed rupture and a 2.17-fold increase in dextran permeability $(\mathrm{P}<0.05)$, whereas simultaneous exposure to 
A

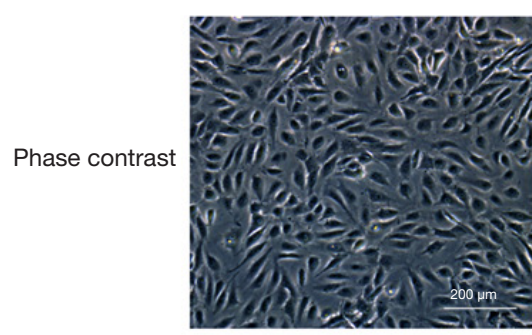

B

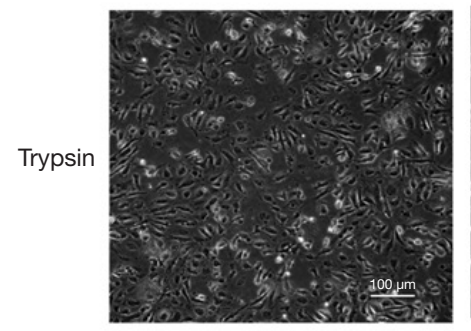

C

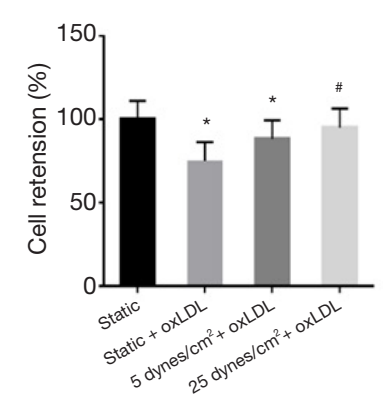

D

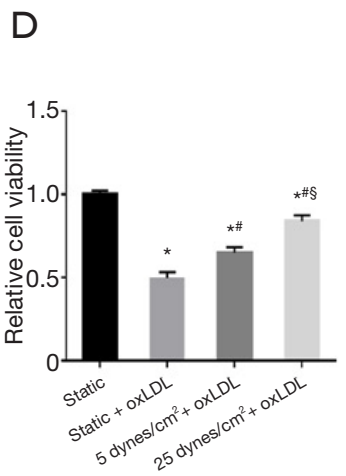

Static + oxLDL
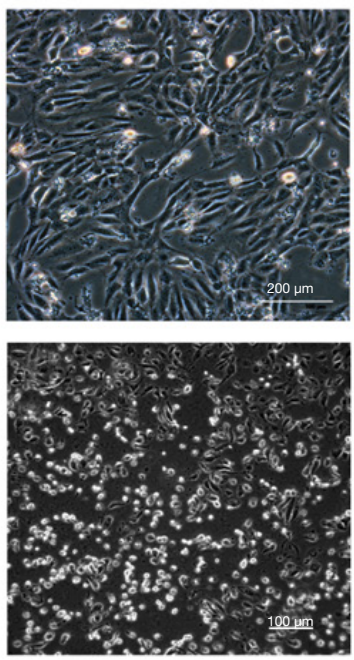

5 dynes $/ \mathrm{cm}^{2}+$ oxLDL
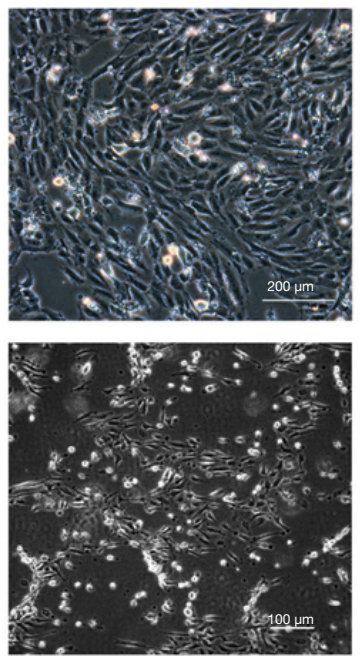

25 dynes $/ \mathrm{cm}^{2}+$ oxLDL
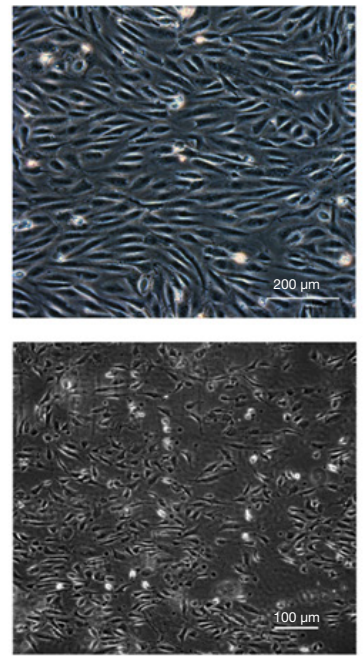

\section{E}

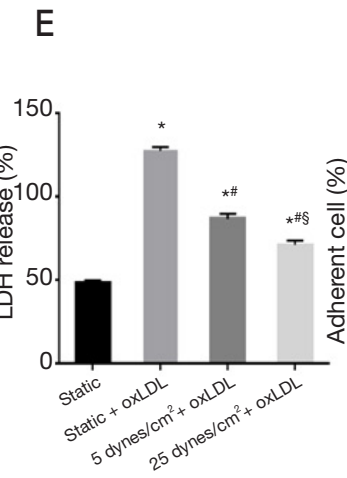

$\mathrm{F}$

Figure 1 Shear Stress affects endothelial cell viability and cell-substrate interactions under atheroprone conditions. (A) Phase-contrast images of the endothelial cells exposed to oxLDL and shear stress $(\times 100)$. Endothelial cells were either subjected to laminar shear stress at 5 or 25 dynes $/ \mathrm{cm}^{2}$ or cultured under static conditions in the presence of oxLDL for 4 hours. (B) Representative phase-contrast images of the endothelial cells after trypsin treatment $(\times 100)$. (C) The retention rate of endothelial cells after shear stress and oxLDL intervention, as seen in introvert microscopy imagines. (D) Cell viability was measured by cell counting kit-8 assay. (E) Cytotoxicity was measured by LDH release levels. (F) Time course displaying number of adherent cells represented as percentage of total cells after trypsinization. Data show mean \pm SEM from 3 in dependent experiments. ${ }^{*}, \mathrm{P}<0.05$ vs. static group; ${ }^{*}$, vs. static + oxLDL group; ${ }^{\S}, v s .5$ dynes $/ \mathrm{cm}^{2}+$ oxLDL group.

oxLDL and 25 dynes $/ \mathrm{cm}^{2}$ LSS led to a $37.79 \%$ decline in the dextran passage $(\mathrm{P}<0.05$ vs. static + oxLDL group) (Figure 2).

The barrier properties of endothelial monolayers were also assessed by TEER measurement using an ECIS system. Under static conditions, endothelial monolayers challenged by oxLDL demonstrated a $28.92 \%$ decrease in TEER compared with non-oxLDL controls $(\mathrm{P}<0.05)$. In contrast, monolayers simultaneously subjected to 25 dynes $/ \mathrm{cm}^{2}$ LSS and oxLDL displayed a $24.97 \%$ increase in TEER compared with the static oxLDL-stimulated endothelial cells $(\mathrm{P}<0.05)$ (Figure 2C,D).
These results indicate that physiologically higher levels of LSS contribute to maintaining the viability and monolayer integrity of endothelial cells and attenuate the oxLDL-induced disruption of the endothelial barrier.

\section{Shear stress alters endothelial cell-cell interactions and reduces vascular endothelial cadherin internalization evoked by oxidized LDL}

Because endothelial permeability depends on the integrity of the VE-cadherin-based adhesion junction, we examined 
A

Static

Static + oxLDL

5 dynes $/ \mathrm{cm}^{2}+$ oxLDL

25 dynes $/ \mathrm{cm}^{2}+$ oxLDL
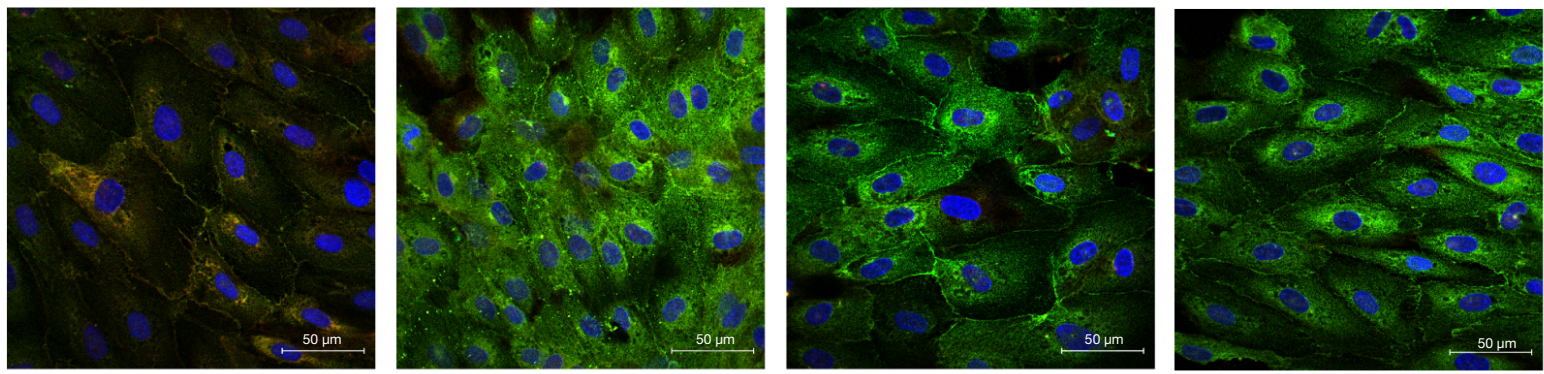

B

C

D
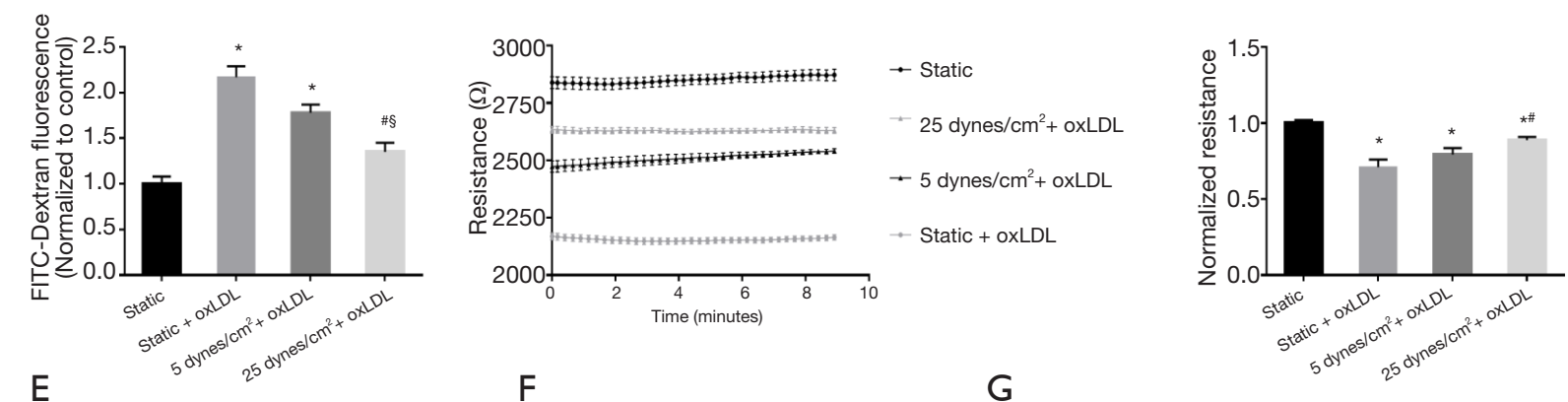

F

G
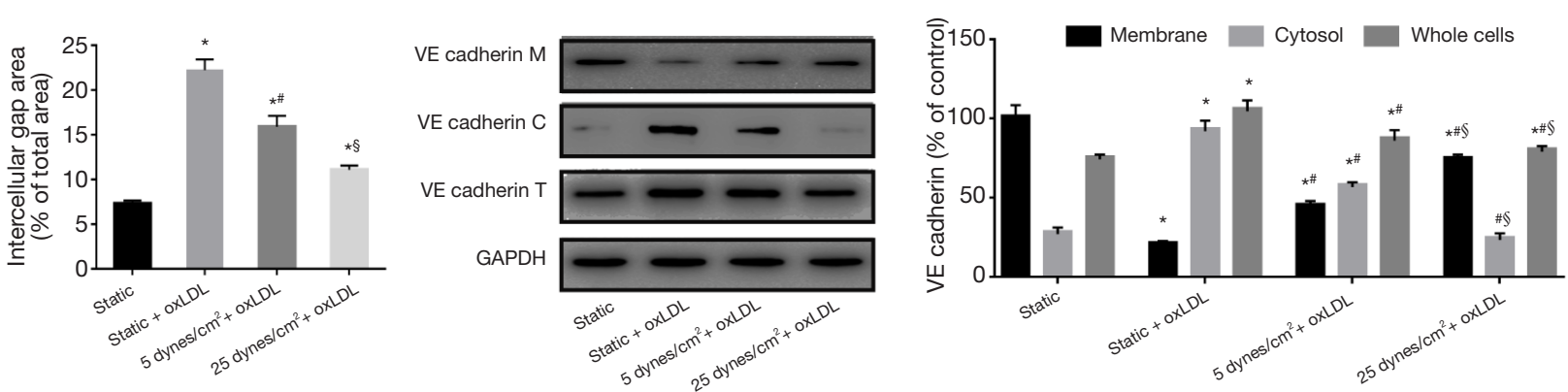

Figure 2 Shear stress affects endothelial integrity and VE-cadherin internalization under atheroprone conditions. (A) Representative confocal laser scanning microscopy images of ECs immunostained for VE-cadherin ( $\times 400)$. (B) Endothelial cell permeability assay by measuring the passage of FITC-dextran through the endothelial monolayer. Less transport of dextran was noticed in endothelial cells exposed to LSS at 25 dynes $/ \mathrm{cm}^{2}$. Data show mean \pm SEM of relative endothelial cell permeability (normalized to control cells) from 6 independent experiments. (C) An illustrative example from a single experiment showing endothelial TEER. TEER reduction induced by oxLDL was reversed by elevated shear stress. (D) Normalized resistance of endothelial monolayers measured by the ECIS system. Graph displays mean data from five independent experiments presented as a percentage change from baseline. (E) Quantification of interendothelial gap areas by immunofluorescence staining of VE-cadherin. (F,G) Representative Western blots and quantitative data were showing the expression of VEcadherin in the membrane (upper panel), cytosol (second panel), and whole cells (third panel), normalized to GAPDH expression. Data show mean \pm SEM from 3 independent experiments. * $\mathrm{P}<0.05$ vs. static group; ${ }^{*}$, vs. static + oxLDL group; ${ }^{\S}, v s .5$ dynes $/ \mathrm{cm}^{2}+$ oxLDL group. 
the expression and distribution of VE-cadherin in endothelial cells. Under static conditions, VE-cadherin fluorescence staining appeared as continuous light lines along the border of the endothelial cells and was also expressed in the cytoplasm. The oxLDL challenge resulted in significantly enhanced VE-cadherin immunostaining, but was mainly located in the cytoplasm, whereas membrane immunostaining was interrupted and weakened (Figure 2A). Western blot analysis showed that compared with the static control group, the cytoplasmic fraction of static oxLDLstimulated endothelial cells increased by 2.40 -fold, and the membrane fraction decreased by $80.08 \%$, indicating VEcadherin turnover $(\mathrm{P}<0.05)$ (Figure $2 F, G)$. In line with these results, a 2.04-fold increase in intercellular gap formation was observed in these static oxLDL-stimulated endothelial cells ( $\mathrm{P}<0.05$ vs. static group) (Figure $2 E)$.

Immunofluorescence staining of $\mathrm{VE}$-cadherin in the endothelial monolayer under oxLDL plus 25 dynes $/ \mathrm{cm}^{2}$ LSS showed an increase in membrane localization of VEcadherin, accompanied by a 2.7 -fold increase in membrane fraction and a noticeable decrease in cytoplasmic and total cellular content (Figure $2 F, G$ ). The intercellular gap area was also reduced by $50.05 \%$ compared to static oxLDLstimulated endothelial cells $(\mathrm{P}<0.05)$ (Figure $2 E)$.

These results suggest the role of physiologically higher levels of LSS in protecting cell-cell interactions and the availability of VE-cadherin on the endothelial cell surface under atheroprone conditions.

\section{Shear stress affects endothelial cell-substrate interactions and attenuates focal adbesion remodeling evoked by oxidized LDL}

After the intervention above, endothelial cells were trypsinized, and the detachment process was followed. The oxLDL treatment significantly accelerated the detachment of static cells, which could be observed as early as 1 minute after trypsin digestion, and was still obvious at later time points. After 5 minutes, the detachment rate was $56.54 \%$ (Figure 1B,F). Of the endothelial cells exposed to 25 dynes $/ \mathrm{cm}^{2}$ LSS plus oxLDL, only $38.52 \%$ were shed, displaying an evident resistance to trypsin $(\mathrm{P}<0.05$ vs. static + oxLDL group) and relatively strong cell-substrate adhesion.

FA consists of a large number of proteins, of which integrins, focal adhesion kinase (FAK), vinculin, and paxillin are the main components. Since the growth or maturation of FA is reflected by size and location, we observed the expression and distribution of vinculin-labelled FA in endothelial cells. We classified FA into three groups: small (nascent, $0.1-0.3 \mu \mathrm{m}^{2}$ ), intermediate $\left(0.3-1 \mu \mathrm{m}^{2}\right)$, large (mature, $1-3 \mu \mathrm{m}^{2}$ and supermature, $>3 \mu \mathrm{m}^{2}$ ), as described previously (19). Immunofluorescence image revealed unique plaque-like positive staining of vinculin around the nonoxLDL static cells, represented a relatively mature form of FA. After oxLDL stimulation, the fluorescence staining of vinculin was much more pronounced, but mainly in the cytoplasm, not on the cell membrane. Peripheral plaquelike staining was difficult to find in the static oxLDL-treated group (Figure 3A). Upon closer inspection, it was found that these strong positives consisted of numerous small dots that occupied most of the cytoplasm and highlighted deformed cells. Quantitative analysis showed that, compared to the untreated control, oxLDL stimulation significantly reduced the size of adhesions and increased the number of small FAs $\left(<0.3 \mu \mathrm{m}^{2}\right)$ by 2.78 -fold, whereas the proportion of large $\mathrm{FA}\left(>1 \mathrm{\mu m}^{2}\right)$ decreased $(\mathrm{P}<0.05)$ (Figure $\left.3 B, C\right)$. These data suggest a reduction in the maturity of FA in oxLDL-evoked cells under static conditions. However, this situation improved when the VEC were subjected to shear stress. Peripheral plaque-like staining reappeared, and the fluorescence staining of vinculin became less prominent. This change was more pronounced in the $25 \mathrm{dyne} / \mathrm{cm}^{2}+$ oxLDL group compared to the 5 dyne $/ \mathrm{cm}^{2}+$ oxLDL group. The average FA size in the 5 dyne/ $\mathrm{cm}^{2}+$ oxLDL group appeared to be larger than the static oxLDL-stimulated endothelial cells but did not reach statistical significance, whereas the simultaneous application of 25 dynes $/ \mathrm{cm}^{2}$ LSS and oxLDL resulted in fewer but larger FAs. Small adhesions $\left(<0.3 \mu \mathrm{m}^{2}\right)$ in the $25 \mathrm{dyne} / \mathrm{cm}^{2}+$ oxLDL group decreased by $49.73 \%$, and large FAs $\left(>3 \mu \mathrm{m}^{2}\right)$ increased by $45.22 \%$ compared with the static oxLDL group. As a result, the average FA size was 1.26 times larger than that of static cells stimulated by oxLDL (Figure 3B,C).

Consistent with the morphologic changes of FA, the results of Western blot analysis further confirmed this trend. Under static conditions, compared with nonoxLDL-treated cells, oxLDL-treated VECs displayed a $60 \%$ reduction in vinculin membrane fractions, a 2.09-fold increase in cytosol content, and a 2.23 -fold increase in total cell lysate $(\mathrm{P}<0.05)$ (Figure 3D,E). Shear stress exposure increased vinculin membrane fractions and decreased cytoplasm and total cell content in both the 5 dynes $/ \mathrm{cm}^{2}$ + oxLDL group and the 25 dynes $/ \mathrm{cm}^{2}+$ oxLDL group, and the membrane and total cell fraction changes in the 25 dynes $/ \mathrm{cm}^{2}+$ oxLDL group were greater than the 5 dynes $/ \mathrm{cm}^{2}+$ oxLDL group $(\mathrm{P}<0.05)$ (Figure $\left.3 D, E\right)$. 
A Static

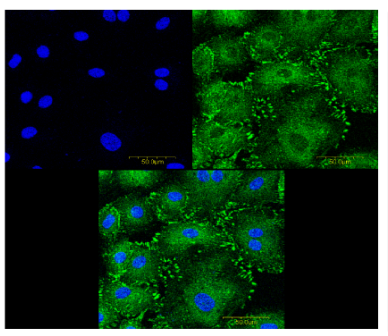

B

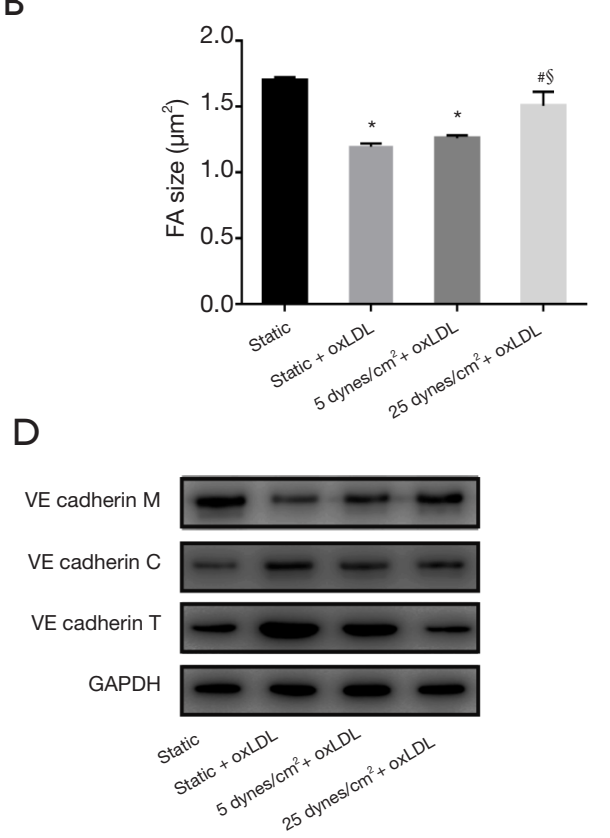

Static + oxLDL
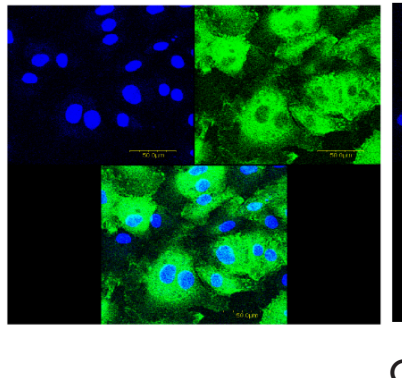

c
5 dynes $/ \mathrm{cm}^{2}+$ oxLDL

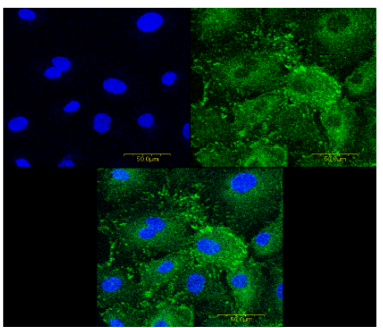

25 dynes $/ \mathrm{cm}^{2}+$ oxLDL
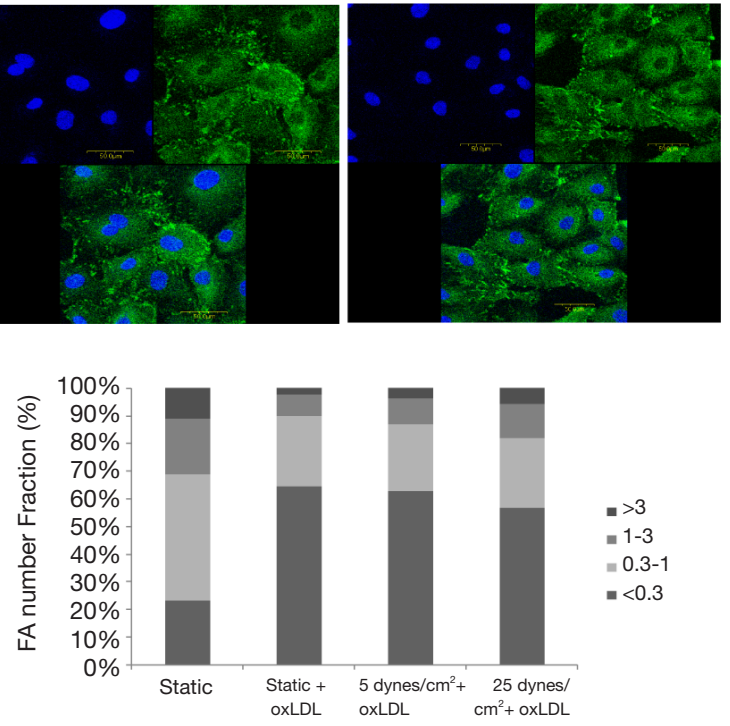

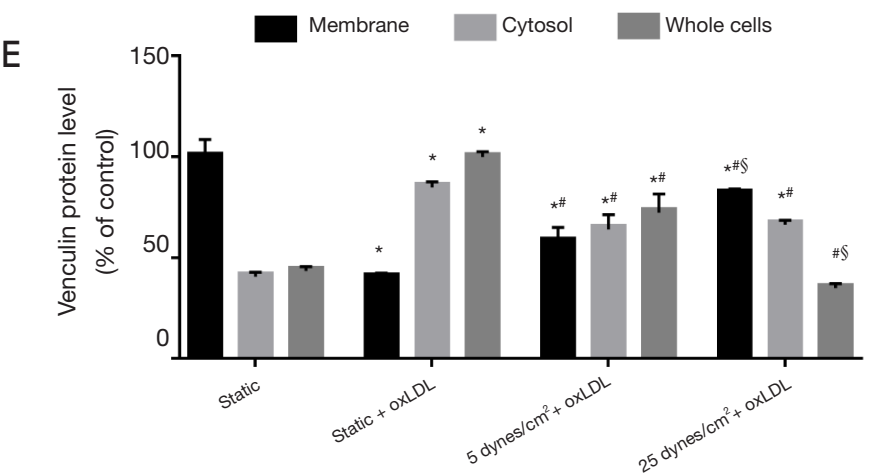

Figure 3 Shear Stress affects focal adhesion size, number, and location of endothelial cells under atheroprone conditions. (A) Representative confocal laser scanning microscope images of ECs immunostained for vinculin ( $\times 400)$. (B) Vinculin- labelled adhesion size was assessed using ImageJ. (C) Number fraction of vinculin- containing focal adhesion with a defined size. (D,E) Representative Western blots and quantitative data were showing the expression of vinculin in the membrane (upper panel), cytosol (second panel), and whole cells (third panel), normalized to GAPDH expression. Data show mean \pm SEM from 3 independent experiments. * $\mathrm{P}<0.05$ vs. static group; ${ }^{\text {, }}$ vs. static + oxLDL group; ${ }^{\S}$, vs. 5 dynes $/ \mathrm{cm}^{2}+$ oxLDL group.

To further investigate the effect of shear stress on more FA components, immunofluorescence staining was used to observe the expression of integrin $\beta 1$ and FAK (Figure $4 A, B$ ). The OxLDL challenge produced diffuse and prominent integrin $\beta 1$ and FAK in static cells, and the simultaneous application of 25 dynes $/ \mathrm{cm}^{2}$ LSS and oxLDL down-regulated the expression of integrin $\beta 1$ and FAK. These trends were further confirmed by flow cytometry or Western blot analysis $(\mathrm{P}<0.05)$ (Figure $4 C, D, E)$.

Collectively, our results suggest that physiological higher levels of LSS result in enhanced cell attachment, promote FA maturity, and attenuate oxLDL-induced vinculin internalization.

\section{Shear stress decreases monocyte adbesion and transendothelial migration evoked by oxidized LDL}

The transendothelial migration of leukocytes is a critical event in inflammation and atherosclerosis. VE-cadherin gap formation and endothelial barrier disruption are 
A

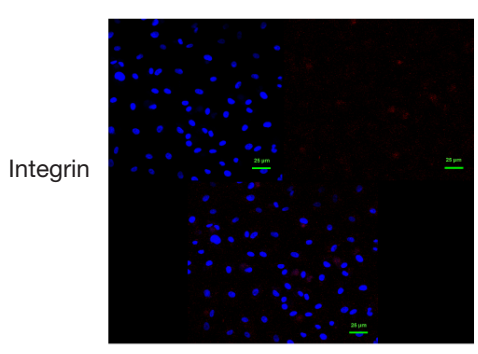

B

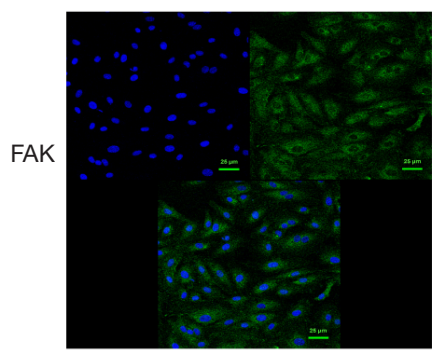

C

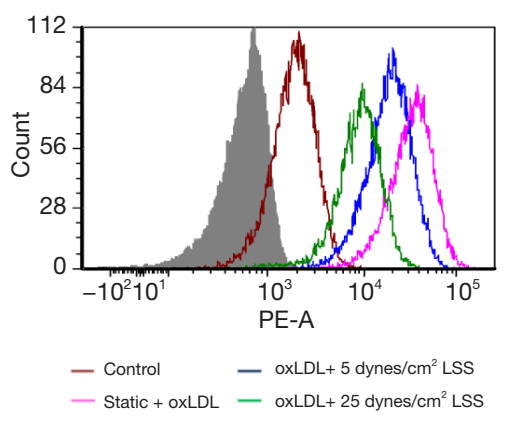

Static + oxLDL
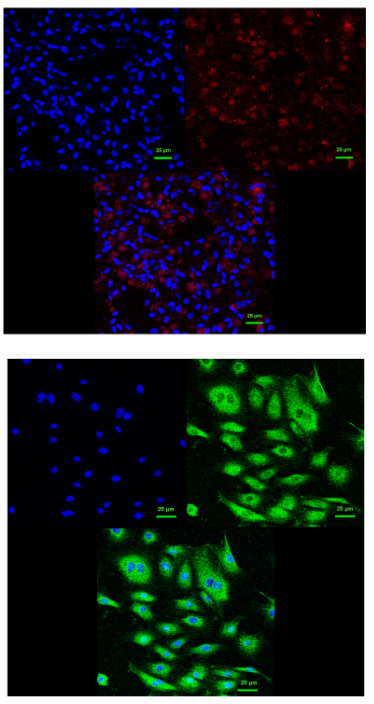

$\mathrm{D}$

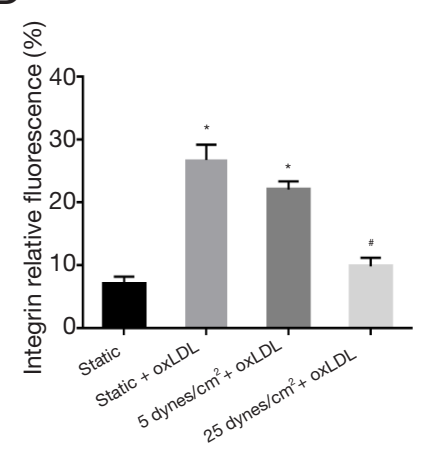

5 dynes $/ \mathrm{cm}^{2}+$ oxLDL
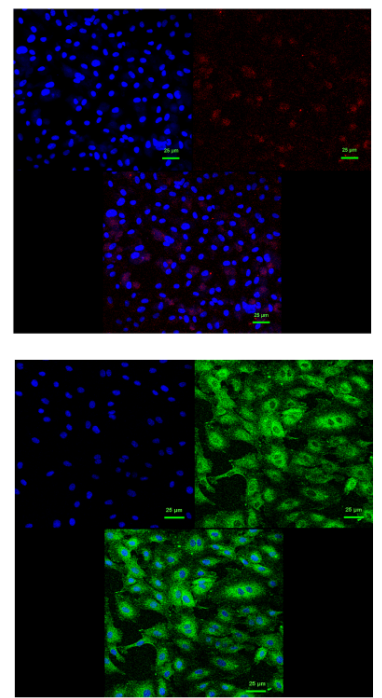

25 dynes $/ \mathrm{cm}^{2}+$ oxLDL
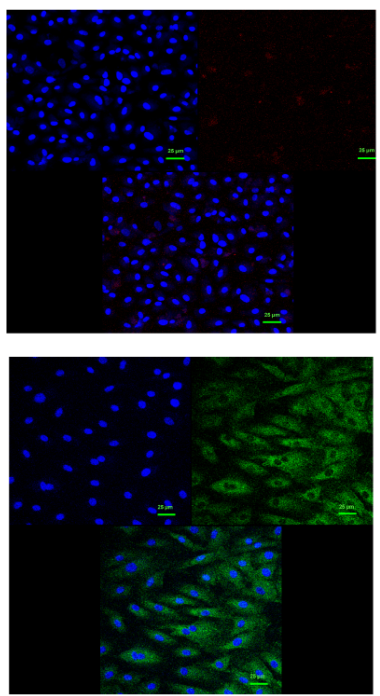

E GAPDH
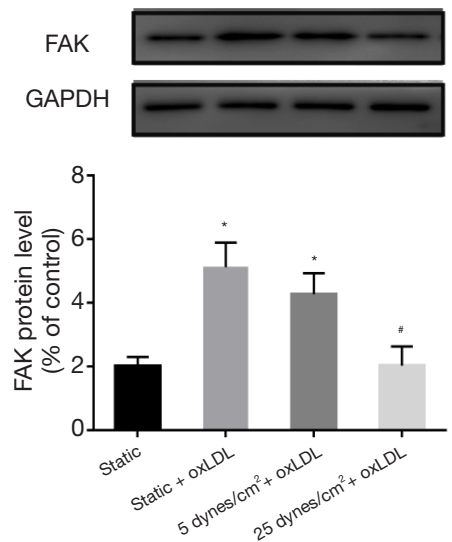

Figure 4 Shear stress affects focal adhesion remodeling of endothelial cells under atheroprone conditions. (A) Immunofluorescence confocal laser scanning microscope images of HUVEC stained with integrin $\beta 1$ ( $\times 200)$. (B) Immunofluorescence microscopy images of HUVEC stained with FAK antibody. Colors indicate positive staining for integrin $\beta 1$ (red), FAK (green) and cell nuclei (blue) ( $\times 200)$ ). (C,D) Flow cytometry assessment of integrin $\beta 1$. (E) Western blot bands and density quantification of FAK. Data show mean \pm SEM from 5 independent experiments. *, $\mathrm{P}<0.05$ vs. static group; ${ }^{*}$, vs. static + oxLDL group; ${ }^{\S}$, vs. 5 dynes $/ \mathrm{cm}^{2}+$ oxLDL group.

required for leukocyte transmigration. Therefore, we investigated the combined impact of shear stress and oxLDL on the resistance of the endothelial barrier to monocytes in a flow chamber. Following the intervention described earlier, BCECF-AM-labeled quiescent THP1 cells were plated on the endothelial monolayer and incubated. Under static conditions, oxLDL stimulation resulted in a remarkable increase in THP-1 adhesion to endothelial monolayers (Figure 5). By measuring the fluorescence intensity of THP-1 cells that migrated from the lower chamber through the endothelial monolayer to the upper chamber, we found that oxLDL increased the migration of THP- 1 cells by 1.82 -fold $(\mathrm{P}<0.05$ vs. static group) (Figure $5 E$ ) under static conditions, accompanied by VCAM-1 and MCP-1 enhancement $(\mathrm{P}<0.05)$ (Figure $5 C, F, G, H)$. The simultaneous exposure of endothelial cells to 5 dyne $/ \mathrm{cm}^{2}$ LSS and oxLDL could alleviate this effect. The simultaneous exposure to $25 \mathrm{dyne} / \mathrm{cm}^{2}$ LSS and oxLDL could further alleviate this effect by reducing the adhesion and migration of THP-1 cells $(62.83 \%$ and $57.28 \%$, respectively, $\mathrm{P}<0.05$ vs. static + oxLDL group) (Figure $5 A, B, D, E)$. The expression levels of VCAM-1 and 
A

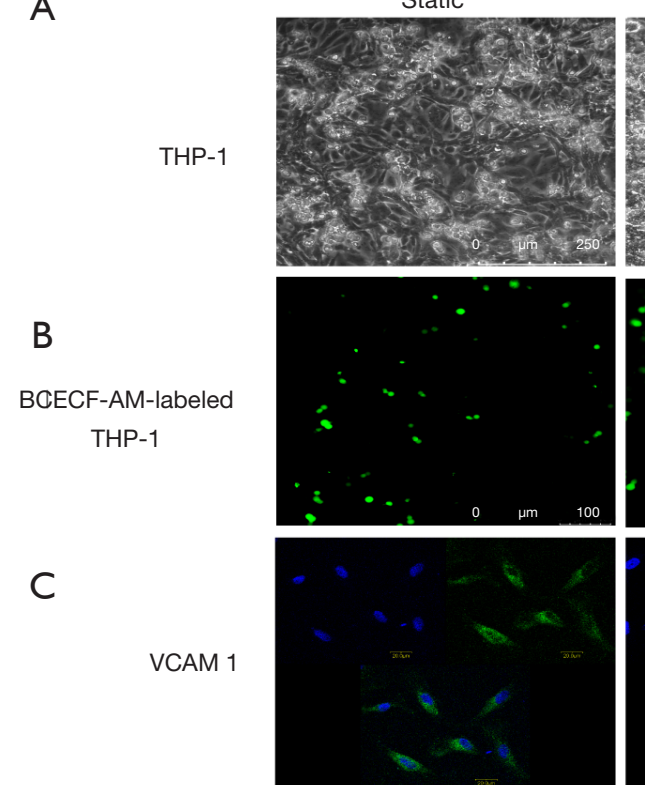

Static + oxLDL
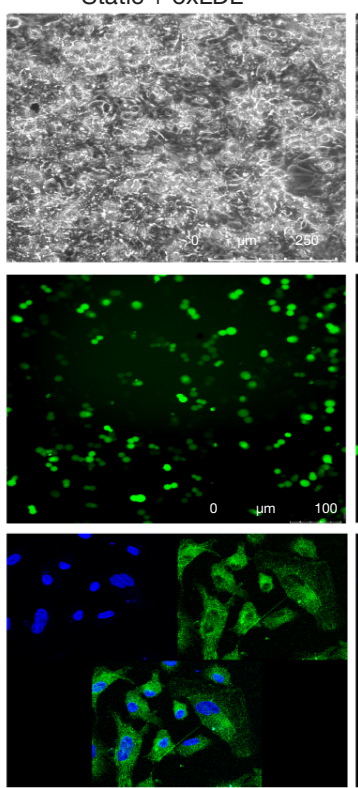

5 dynes $/ \mathrm{cm}^{2}+$ oxLDL

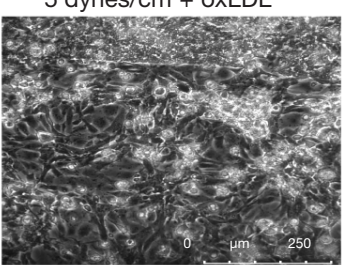

25 dynes $/ \mathrm{cm}^{2}+$ oxLDL
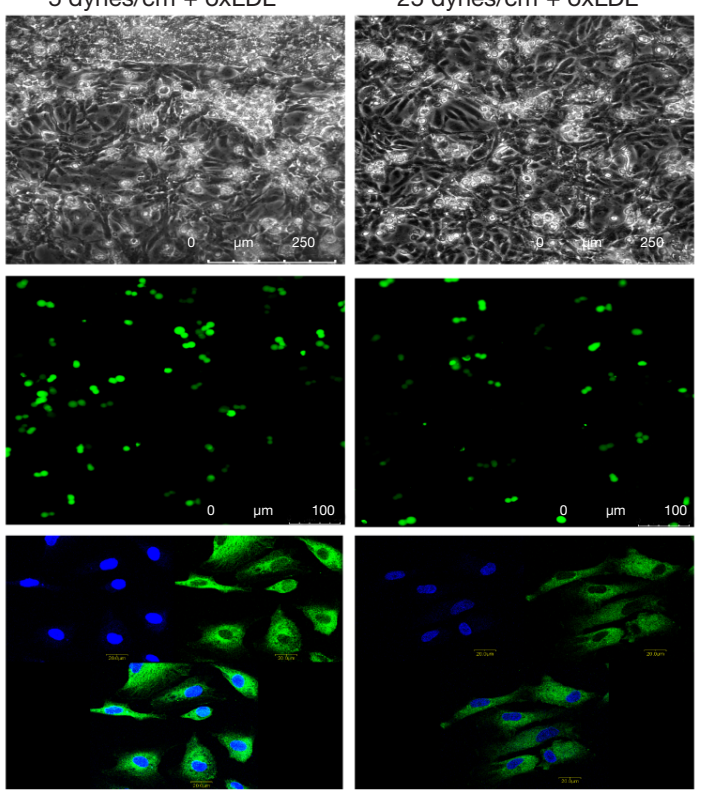

D

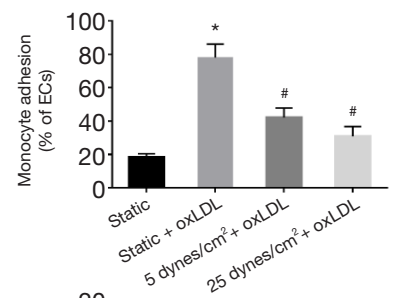

$\mathrm{F}$

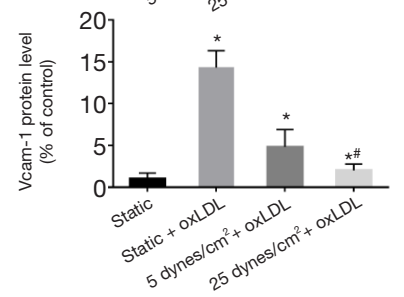

E

G
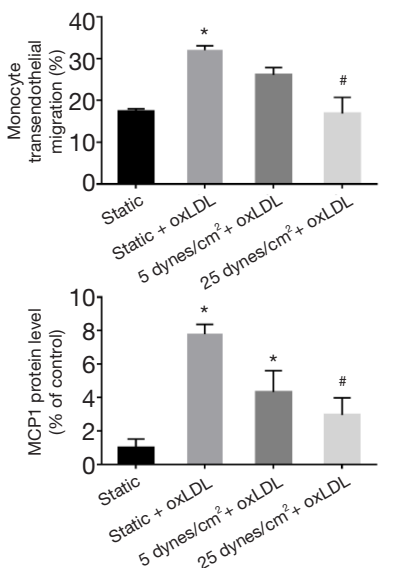

$\mathrm{H}$

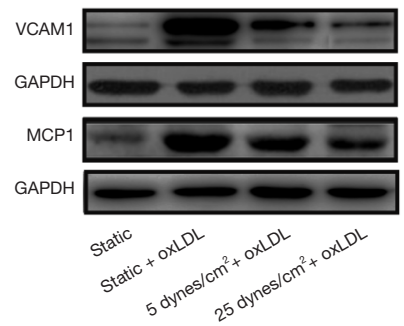

Figure 5 Shear stress affects monocyte/endothelial cell adhesion and transendothelial migration under atheroprone conditions. (A) Representative phase-contrast images of THP-1 monocyte adhesion to endothelial monolayer after the intervention $(\times 400)$. (B) Representative immunofluorescence microscopy images of BCECF-AM-labeled THP-1 monocyte adhesion to endothelial monolayer (×200). (C) Immunofluorescence microscopic images of HUVEC stained with VCAM-1 antibody ( $\times 600)$. Colors indicate positive staining for VCAM-1 (green), and cell nuclei (blue). (D) quantification of THP-1 monocyte adhesion to endothelial monolayer. (E) Quantification of the transmigration of THP-1 cells through endothelial monolayers by measuring the fluorescence intensity. (F,G,H) Western blot bands and density quantification of VCAM-1 and MCP-1. Data show mean \pm SEM from 5 independent experiments. *, $\mathrm{P}<0.05$ vs. static group; *, $v s$. static + oxLDL group; ${ }^{\S}, v s .5$ dynes $/ \mathrm{cm}^{2}+$ oxLDL group.

MCP-1 were also reduced in endothelial cells receiving 25 dyne $/ \mathrm{cm}^{2}$ LSS plus oxLDL $(\mathrm{P}<0.05$ vs. static + oxLDL group) (Figure 5C,F,G,H).

These results indicate that physiologically higher levels of LSS attenuate oxLDL-induced monocyte/endothelial cell adhesion and transmigration, as well as the overexpression of VCAM-1 and MCP-1.

\section{Discussion}

VECs lining the blood vessels are continuously subjected to shear stress and cyclic stress from the blood flow. Following the transduction of extracellular signals at FA and cadherin complexes, intracellular signaling cascades reach the nucleus to active genes that respond to mechanical forces, which 
allow arteries to adapt to their physical environment (24). The phenotype of endothelial cells exposed to LSS $>10$ dynes $/ \mathrm{cm}^{2}$ is characterized by enhanced endothelial nitric oxide (NO) synthase activity and NO bioavailability; prolonged endothelial cell survival, and an anticoagulant, anti-adhesive cell surface $(25,26)$. It was reported that individuals who engage in regular exercise and physical activity have an average life expectancy approximately 7 years longer than their sedentary counterparts. Exercise is a powerful therapy for slowing the progression of cardiovascular disease. One of its essential mechanisms is related to the increase in blood flow and shear stress affecting the phenotype of VECs $(27,28)$.

The vascular endothelium works as a semi-permeable barrier between the bloodstream and the vascular wall. The stability of the endothelial barrier depends on the adhesion between adjacent endothelial cells and also the adhesion of endothelial cells to the underlying extracellular matrix. The intercellular adhesion is mainly composed of VE-cadherin (29,30). In most types of cells, the cellular contact point with the extracellular matrix is known as the FA. FAs act as ideal checkpoints controlling the inwards and inside-out transduction of molecular signals. This signaling function is critical for endothelial cells to respond to mechanical stress, inflammation, and atheroprone factors, also required for the alignment of endothelial cells along the flow direction. The size of FA depends on the stage of maturity and cell type. Endothelial nascent FAs have an average size of approximately $0.2 \mathrm{~m}^{2}$, they can grow and mature into intermediate-sized FAs $\left(0.5-1 \mathrm{~m}^{2}\right)$ and then become large FAs, usually greater than $1 \mu^{2}$ (31,32). Our research adds to evidence that, in the case of atherosclerosis evoked by oxLDL, the number of $\mathrm{FA}$ increases, but the size of $\mathrm{FA}$, especially large, mature FA decreases, leading to FA dysfunction, and eventually the detachment of endothelial cells from the matrix.

Studies investigating the role of FAK in vascular endothelial integrity have had different results. Several groups have shown that FAK activation enhances endothelial barrier function, whereas others report that FAK activation leads to endothelial barrier dysfunction (33-35). In the present study, oxLDL-induced endothelial barrier disruption was related to the overexpression of $\mathrm{VE}$-cadherin and FA components (including integrin, FAK and vinculin), and their location changed from the cell membrane into cytoplasm. The internalization of VE-cadherin and vinculin represents the abnormal remodeling of FA. These effects are particularly noticeable under static conditions and can be attenuated by the exposure of EC to physiological shear stress. Elevated shear stress contributes to the redistribution of $\mathrm{VE}$-cadherin and vinculin to the periphery of cell, which may be their true functional status.

Endothelial cells undergo phenotypic changes when stimulated by pro-atherogenic factors. The initial step is the disruption of endothelial junctions resulting in increased paracellular permeability, also known as type I endothelial cell activation. During subsequent type II endothelial activation, expression of inflammatory and adhesion molecules is triggered, leading to monocytes/ macrophages recruitment and migration. A vital function of the endothelium is to act as a barrier to prevent monocyte adhesion and subsequent infiltration $(36,37)$. Our study provides additional evidence that the exposure of endothelial cells to physiological LSS helps inhibit monocyte adhesion and transmigration, highlighting the beneficial response of the endothelium to atheroprotective flow.

One limitation of the present study was that the molecular mechanism responsible for shear stress transduction in VEC has not been fully established. Another limitation was the relatively short exposure time to shear stress. Although a shear force of $25 \mathrm{dyne} / \mathrm{cm}^{2}$ for 4 hours is sufficient to impact endothelial morphology and function, if the loading time is prolonged, the difference between the groups may be more significant.

However, it should be also taken into account that excessive mechanical forces may be harmful to endothelial cells and even cause the rupture of vulnerable plaques, leading to acute cardio-cerebral vascular events. It has reported that plaque rupture most often occurs at a proximal location to the site of maximal stenosis of an artery, and upstream of the plaque, which is the region with steeply increasing wall shear stress usually above 40 dynes $/ \mathrm{cm}^{2}$ (38). Therefore, we need to keep in mind the risk of excessive shear stress in advanced atherosclerosis. In our experimental model, shear stress applied to endothelial monolayer was within the physiological range, and oxLDL and shear stress are simultaneously loaded onto endothelial cells to mimic the early stage of atherosclerosis. Our results highlight the role of shear stress in protecting endothelial barrier under these atheroprone conditions. Intervention strategy targeting appropriate elevation in shear stress can be used as therapeutic approaches to prevent and treat atherosclerotic diseases.

\section{Acknowledgments}

Funding: The present study was supported by the National 


\section{Page 12 of 13}

Natural Science Funds of China (no. 30871047), the Funds of Guangdong Provincial Project of Science and Technology (no. 2013B021800294).

\section{Footnote}

Data Sharing Statement: Available at http://dx.doi. org/10.21037/atm-20-3426

Peer Review File: Available at http://dx.doi.org/10.21037/atm20-3426

Conflicts of Interest: All authors have completed the ICMJE uniform disclosure form (available at http://dx.doi. org/10.21037/atm-20-3426). The authors have no conflicts of interest to declare.

Ethical Statement: The authors are accountable for all aspects of the work in ensuring that questions related to the accuracy or integrity of any part of the work are appropriately investigated and resolved. The study was conducted in accordance with the Declaration of Helsinki (as was revised in 2013). The study was approved by the Ethics Committee of the First Affiliated Hospital, Sun Yat-sen University, China (No. 201060), and informed consent was taken from all the participants.

Open Access Statement: This is an Open Access article distributed in accordance with the Creative Commons Attribution-NonCommercial-NoDerivs 4.0 International License (CC BY-NC-ND 4.0), which permits the noncommercial replication and distribution of the article with the strict proviso that no changes or edits are made and the original work is properly cited (including links to both the formal publication through the relevant DOI and the license). See: https://creativecommons.org/licenses/by-nc-nd/4.0/.

\section{References}

1. Poznyak AV, Wu WK, Melnichenko AA, et al. Signaling pathways and key genes involved in regulation of foam cell formation in atherosclerosis. Cells 2020;9:584.

2. Hartley A, Haskard D, Khamis R. Oxidized LDL and antioxidized LDL antibodies in atherosclerosis-Novel insights and future directions in diagnosis and therapy. Trends Cardiovasc Med 2019; 29:22-6.

3. Mundi S, Massaro M, Scoditti E, et al. Endothelial permeability, LDL deposition, and cardiovascular risk
Zhong et al. Endothelial barrier modulation under flow and oxLDL

factors- a review. Cardiovasc Res 2018;114:35-52.

4. Wang Q, Wang Y, Lehto K, et al. Genetically-predicted life-long lowering of low-density lipoprotein cholesterol is associated with decreased frailty: A Mendelian randomization study in UK biobank. EBioMedicine 2019;45:487-94.

5. Markin AM, Sobenin IA, Grechko AV, et al. Mechanisms of human atherogenesis: focus on chronification of inflammation and mitochondrial mutations. Front Pharmacol 2020;11:642.

6. Malek AM, Alper SL, Izumo S. Hemodynamic shear stress and its role in atherosclerosis. JAMA 1999;282:2035-2042.

7. Zhang Y, He X, Liu D, et al. Enhanced External Counterpulsation attenuates atherosclerosis progression through modulation of proinflammatory signal pathway. Arterioscler Thromb Vasc Biol 2010;30:773-80.

8. Xu S, Koroleva M, Yin M, et al. Atheroprotective laminar flow inhibits Hippo pathway effector YAP in endothelial cells. Transl Res 2016;176:18-28.e2.

9. Greyling A, Schreuder TH, Landman T, et al. Elevation in blood flow and shear rate prevents hyperglycemia-induced endothelial dysfunction in healthy subjects and those with type 2 diabetes. J Appl Physiol (1985) 2015;118:579-85 .

10. Warboys CM, Eric Berson R, Mann GE, et al. Acute and chronic exposure to shear stress has opposite effects on endothelial permeability to macromolecules. Am J Physiol Heart Circ Physiol 2010;298:H1850-6.

11. Jiang H, Huang S, Li X, et al. Tyrosine Kinase Receptor $\mathrm{B}$ protects against coronary artery disease and promotes adult vasculature integrity by regulating Ets1-mediated VE-Cadherin expression. Arterioscler Thromb Vasc Biol 2015;35:580-8.

12. Zhao X, Peng X, Sun S, et al. Role of kinase-independent and-dependent functions of FAK in endothelial cell survival and barrier function during embryonic development. J Cell Biol 2010;189:955-65.

13. Puleo JI, Parker SS, Roman MR, et al. Mechanosensing during directed cell migration requires dynamic actin polymerization at focal adhesions. J Cell Biol 2019;218:4215-35.

14. Baudin B, Bruneel A, Bosselut N, et al. A protocol for isolation and culture of human umbilical vein endothelial cells. Nat Protoc 2007;2:481-5.

15. Moonen JR, Lee ES, Schmidt M, et al. Endothelial-tomesenchymal transition contributes to fibro-proliferative vascular disease and is modulated by fluid shear stress. Cardiovasc Res 2015;108:377-86.

16. Mahmoud MM, Kim HR, Xing R, et al. TWIST1 integrates 
endothelial responses to flow in vascular dysfunction and atherosclerosis. Circ Res 2016;119:450-62.

17. Zhang Y, Song H, Zhang Y, et al. Irisin inhibits atherosclerosis by promoting endothelial proliferation through microRNA126-5p. J Am Heart Assoc 2016;5:e004031.

18. Szulcek R, Bogaard HJ, van Nieuw Amerongen GP. Electric cell- substrate impedance sensing for the quantification of endothelial proliferation, barrier function, and motility. J Vis Exp 2014. doi: 10.3791/51300.

19. Bevan HS, Slater SC, Clarke H, et al. Acute laminar shear stress reversibly increases human glomerular endothelial cell permeability via activation of endothelial nitric oxide synthase. Am J Physiol Renal Physiol 2011;301:F733-42.

20. Goetsch KP, Snyman C, Myburgh KH, et al. Rock-2 is associated with focal adhesion maturation during myoblast migration. J Cell Biochem 2014;115:1299-307.

21. Chen PW, Jian X, Yoon HY, et al. ARAP2 signals through Arf6 and Rac1 to control focal adhesion morphology. J Biol Chem 2013;288:5849-60.

22. Walpen T, Peier M, Haas E, et al. Loss of Pim1 imposes a hyperadhesive phenotype on endothelial cells. Cell Physiol Biochem 2012;30:1083-96.

23. Zhang Y, He X, Chen X, et al. Enhanced External Counterpulsation inhibits intimal hyperplasia by modifying shear stress responsive gene expression in hypercholesterolemic pigs. Circulation 2007;116:526-34.

24. Ghimire K, Zaric J, Alday-Parejo B, et al. MAGI1 mediates eNOS activation and NO production in endothelial cells in response to fluid shear stress. Cells 2019. doi: 10.3390/ cells8050388.

25. Ding SF, Ni M, Liu XL, et al. A causal relationship between shear stress and atherosclerotic lesions in apolipoprotein E knockout mice assessed by ultrasound biomicroscopy. Am J Physiol Heart Circ Physiol 2010;298:H2121-9.

26. Davies PF, Civelek M, Fang Y, et al. The atherosusceptible endothelium: endothelial phenotypes in complex haemodynamic shear stress regions in vivo. Cardiovasc Res 2013;99:315-27.

27. Parsons C, Agasthi P, Mookadam F, et al. Reversal of coronary atherosclerosis: Role of life style and medical management. Trends Cardiovasc Med 2018;28:524-31.

28. Grimm H, Kretzschmar J, Cook MD, et al. The effects of exercise, Aspirin, and Celecoxib in an atherogenic environment. Med Sci Sports Exerc 2018;50:2033-9.
29. Chrifi I, Louzao-Martinez L, Brandt M, et al. CMTM3 (CKLF-Like Marvel Transmembrane Domain 3) mediates angiogenesis by regulating cell surface availability of $\mathrm{VE}$ Cadherin in endothelial adherens junctions. Arterioscler Thromb Vasc Biol 2017;37:1098-114.

30. Andresen Eguiluz RC, Kaylan KB, Underhill GH, et al. Substrate stiffness and VE-cadherin mechano-transduction coordinate to regulate endothelial monolayer integrity. Biomaterials 2017;140:45-57.

31. Cleghorn WM, Bulus N, Kook S, et al. Non-visual arrestins regulate the focal adhesion formation via small GTPases RhoA and Rac1 independently of GPCRs. Cell Signal 2018;42:259-69.

32. Israeli-Rosenberg S, Manso AM, Okada H, et al. Integrins and integrin-associated proteins in the cardiac myocyte. Circ Res 2014;114:572-86.

33. Zebda N, Dubrovskyi O, Birukov KG. Focal Adhesion Kinase Regulation of mechanotransduction and its impact on endothelial cell functions. Microvasc Res 2012;83:71-81.

34. Infusino GA, Jacobson JR. Endothelial FAK as a therapeutic target in disease. Microvasc Res 2012;83:89-96.

35. Arnold KM, Goeckeler ZM, Wysolmerski RB. Loss of focal adhesion kinase enhances endothelial barrier function and increases focal adhesions. Microcirculation 2013;20:637-49.

36. Lin WL, Chang CF, Shi CS, et al. Recombinant LectinLike Domain of Thrombomodulin suppresses vascular inflammation by reducing leukocyte recruitment via interacting with Lewis $\mathrm{Y}$ on endothelial cells. Arterioscler Thromb Vasc Biol 2013;33:2366-73.

37. Shoulders H, Garner KH, Singla DK. Macrophage depletion by clodronate attenuates bone morphogenetic protein-7induced M2 macrophage differentiation and improved systolic blood velocity in atherosclerosis. Transl Res 2019;203:1-14.

38. Park JB, Choi G, Chun EJ, et al. Computational fluid dynamic measures of wall shear stress are related to coronary lesion characteristics. Heart 2016;102:1655-61.

Cite this article as: Zhong T, Li Y, He X, Liu Y, Dong Y, Ma H, Zheng Z, Zhang Y. Adaptation of endothelial cells to shear stress under atheroprone conditions by modulating internalization of vascular endothelial cadherin and vinculin. Ann Transl Med 2020;8(21):1423. doi: 10.21037/atm-20-3426 